\title{
Cluster and ACE observations of phase synchronization in intermittent magnetic field turbulence: a comparative study of shocked and unshocked solar wind
}

\author{
A. C.-L. Chian and R. A. Miranda \\ National Institute for Space Research (INPE) and World Institute for Space Environment Research (WISER), P.O. Box 515, \\ São José dos Campos-SP 12227-010, Brazil
}

Received: 7 May 2008 - Revised: 23 February 2009 - Accepted: 28 February 2009 - Published: 14 April 2009

\begin{abstract}
We apply two distinct nonlinear techniques, kurtosis and phase coherence index, to analyze the modulus of interplanetary magnetic field data $|B|$ measured by Cluster and ACE spacecraft from 1 to 3 February 2002. High degree of phase synchronization is found across a wide range of time scales, from $1 \mathrm{~s}$ to $10^{4} \mathrm{~s}$, in the magnetic field fluctuations, both in the shocked solar wind upstream of Earth's bow shock and in the unshocked ambient solar wind at the L1 Lagrangian point. This is the first direct measurement of phase coherence in the ambient solar wind turbulence. We show that phase synchronization related to nonlinear multiscale interactions is the origin of the departure from Gaussianity in the intermittent magnetic field turbulence. In particular, we demonstrate that at small scales near the spectral break the intermittency level of Cluster is lower than ACE, which may be a signature of the reflected ions from the shock.
\end{abstract}

Keywords. Interplanetary physics (MHD waves and turbulence; Planetary bow shocks; Solar wind plasma)

\section{Introduction}

The solar wind provides a natural laboratory for observation of intermittent turbulence (Bruno and Carbone, 2005). Nonlinear energy cascade (direct and inverse) due to multiscale interactions leads to localized regions of space plasmas where phase synchronization (phase coherence) involving a finite degree of phase coupling among a number of active modes takes place. Large-amplitude phase coherent (intermittent) structures seen in these localized regions dominate

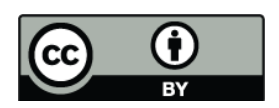

Correspondence to: A. C.-L. Chian (achian@dge.inpe.br) the statistics of fluctuations at small scales and have typical lifetime longer than that of incoherent (random-phase) fluctuations in the background.

A recent theoretical study of nonlinear waves shows that phase synchronization associated with multi-scale interactions is the origin of bursts of coherent structures in intermittent turbulence in plasmas and fluids (He and Chian, 2003, 2005). Observational evidence in support of this finding in space plasma turbulence was obtained by Hada et al. (2003), Koga and Hada (2003) and Koga et al. $(2007,2008)$ using the Geotail solar wind data upstream and downstream of Earth's bow shock, by Sahraoui (2008) using the Cluster data in the magnetosheath close to the Earth's magnetopause, and by Telloni et al. (2009) using the SOHO data of solar corona; and in atmosphere turbulence by Chian et al. (2008) using the Amazon forest data.

The aim of this paper is to seek further observational evidence of phase synchronization in space plasmas based on the magnetic field data of Cluster and ACE (Advanced Composition Explorer) spacecraft. In particular, we compare the phase synchronization detected by Cluster in the magnetic field turbulence in the shocked solar wind upstream of Earth's bow shock with the phase synchronization detected by ACE in the magnetic field turbulence in the unshocked ambient solar wind at the L1 Lagrangian point. Our analysis is performed for the same time interval measured simultaneously by Cluster and ACE from 1 to 3 February 2002. This paper presents the first direct evidence of phase synchronization in the turbulence observed in the ambient solar wind. We focus on the nonlinear analysis of phases and show that the increase of phase synchronization follows the increase of intermittency (kurtosis), which is a demonstration of the close relation between these two processes and a confirmation that intermittency implies "building up" the coherent structures

Published by Copernicus Publications on behalf of the European Geosciences Union. 


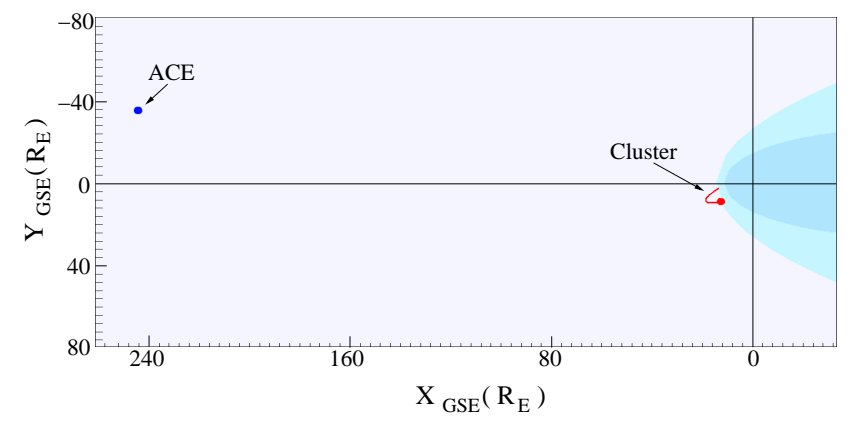

Fig. 1. Orbit trace of Cluster-1 and spacecraft position of ACE, in the GSE coordinate system, from 19:40:40 UT on 1 February 2002 to $03: 56: 38$ UT on 3 February 2002. The starting position of Cluster is shown as a full circle.

(i.e., structures with a finite degree of phase synchronization among a number of scales).

The physical conditions upstream of Earth's bow shock along the path of Cluster are expected to differ from the unshocked ambient solar wind in the vicinity of ACE. The magnetic connection between the interplanetary magnetic field (IMF) and the bow shock may occur sporadically in the upstream solar wind, as evidenced by a strong emission at the local electron plasma frequency (Kellogg and Horbury, 2005). In contrast the ambient solar wind at L1, being far away from the Earth's bow shock, is not affected by the shock. This paper carries out a comparative study of the degree of phase synchronization across a wide range of scales in the interplanetary magnetic field fluctuations in shocked (Cluster) and unshocked (ACE) regions of solar wind.

Cluster has observed intermittent interplanetary turbulence upstream of Earth's bow shock. The first study of solar wind intermittency using Cluster data was reported by Pallocchia et al. (2002). They showed that velocity fluctuations detected by Cluster- 3 are slightly more intermittent than Cluster-1 on 22 February 2001. Bale et al. (2005b) used the Cluster-4 data of 19 February 2002 to show that both electricfield and magnetic-field fluctuations of turbulence in the upstream solar wind display the $k^{-5 / 3}$ spectral behavior of classical Kolmogorov fluid turbulence over an inertial subrange and a spectral break at $k \rho_{i} \sim 0.45$ (where $\rho_{i}$ is the ion Larmor radius). In the dissipative subrange above the spectral break point, the magnetic spectrum becomes steeper while the electric spectrum gets enhanced. They suggest that Alfvén waves in the inertial subrange eventually disperse as kinetic Alfvén waves above the spectral break, becoming more electrostatic at short wavelengths where wave energy is dissipated through wave-particle interaction processes such as Landau or transit time damping. Narita et al. (2006) determined directly the wavenumber power spectra of intermittent magnetic field turbulence in the foreshock of a quasi-parallel bow shock using four-point Cluster spacecraft measurements; they conjectured that nonlinear interactions of Alfvén waves can lead to phase coherence in the foreshock turbulence observed by Cluster. Alexandrova et al. (2007) used the Cluster-1 data of 5 April 2001 to demonstrate that in the inertial subrange below the ion cyclotron frequency, the turbulent spectrum of unshocked solar wind magnetic field follows Kolmogorov's law. However, after the spectral break the turbulence cannot be characterized by a "dissipative" range. Instead, the kurtosis (fourth-order structure function) increases with frequency, similar to the intermittent behavior of the lowfrequency inertial subrange, indicating that nonlinear wave interactions are operating to yield a new high-frequency inertial subrange. Alexandrova et al. (2008) showed that the magnetic field fluctuations within the high-frequency inertial subrange identified by Alexandrova et al. (2007) is much more compressive than the low-frequency inertial subrange dominated by incompressive Alfvén waves. This increase of compressibility is due to a partial dissipation (and destruction of phase coherence) of left-hand Alfvénic fluctuations by the ion cyclotron damping in the neighborhood of the spectral break point around the ion cyclotron frequency, leading to a new right-hand "magnetosonic" small-scale cascade characterized by an increase of intermittency as well as spectrum steepening.

ACE has monitored solar wind in an orbit around the L1 point. Burlaga and Viñas (2004) showed that the fluctuations of solar wind speed observed by ACE are related to intermittent turbulence and shocks at the small scales $(1 \mathrm{~h})$ and can be described by a Tsallis probability distribution function derived from nonextensive statistical mechanics. Smith et al. (2006) demonstrated that while the inertial subrange of solar wind magnetic field turbulence measured by ACE at lower frequencies displays a tightly constrained range of spectral indexes, the dissipation range exhibits a broad range of power-law indexes. Chapman and Hnat (2007) showed that solar wind turbulence detected by ACE is dominated by Alfvénic fluctuations with power spectral exponents that evolve toward the Kolmogorov value of $-5 / 3$, and can be decomposed into two coexistent components perpendicular and parallel to the local average magnetic field. Hamilton et al. (2008) found that on average the wave vectors of solar wind magnetic field turbulence measured by ACE are more field-aligned in the dissipation subrange than in the inertial subrange, and cyclotron damping plays an important but not exclusive role in the formation of the dissipation subrange; moreover, the orientation of the wave vectors for the smallest scales within the inertial subrange are not organized by wind speed and that on average the data shows the same distribution of energy between perpendicular and field-aligned wave vectors.

Recently, a phase coherence technique for characterizing phase synchronization in nonlinear wave-wave coupling and turbulence based on surrogate data has been developed for space plasmas (Hada et al., 2003; Koga and Hada, 2003; Koga et al., 2008; Sahraoui, 2008). The link between phase coherence, non-Gaussianity and intermittent turbulence was 


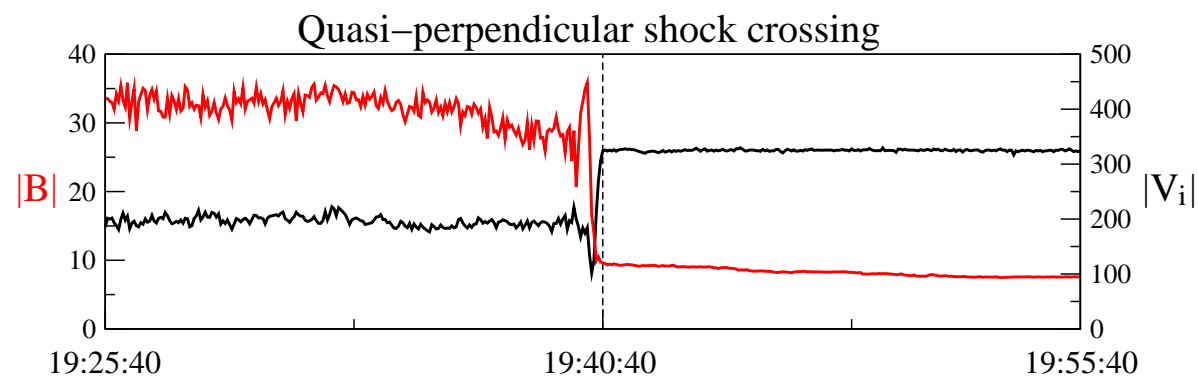

Day 32

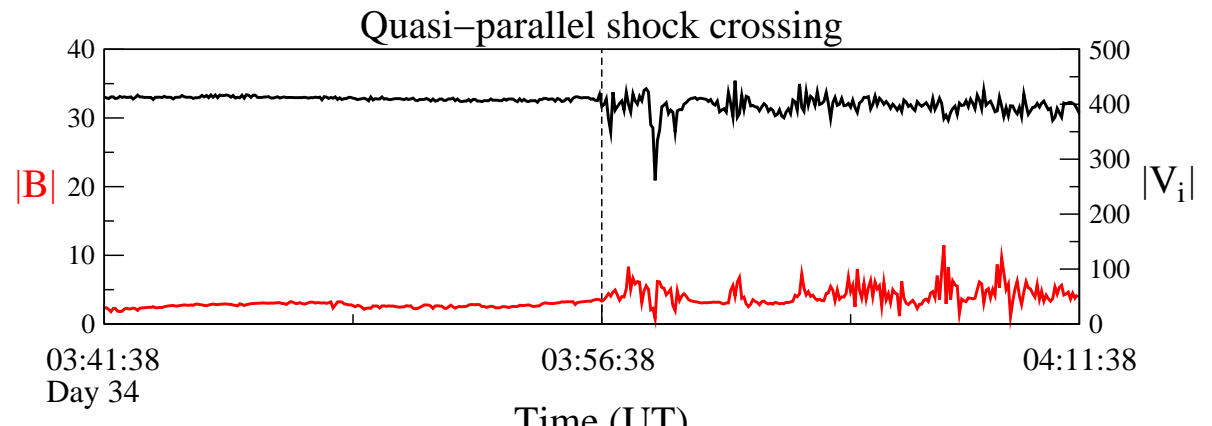

Fig. 2. Cluster-1 magnetic field $|B|$ (red, nT) and ion bulk flow velocity $\left|V_{i}\right|$ (black, $\mathrm{km} / \mathrm{s}$ ) during the quasi-perpendicular shock crossing (upper panel) on Julian day 32, 2002, and the quasi-parallel shock crossing (lower panel) on Julian day 34, 2002. The vertical dashed lines indicate the beginning and the end of the selected time interval of Fig. 3, respectively.

established by Koga et al. (2007), based on the Geotail magnetic field data upstream and downstream of Earth's bow shock. In this paper, we investigate phase synchronization due to nonlinear multiscale interactions and non-Gaussian statistics using the magnetic field data collected by Cluster upstream of Earth's bow shock and by ACE in the ambient solar wind at L1. By applying the phase coherence index technique to quantify the degree of phase synchronization, we show that its variation with time scales is similar to kurtosis indicating a significant departure from Gaussianity over a wide range of time scales, which is enhanced at small scales, in agreement with the leptokurtic shape of small-scale probability density function (PDF) of intermittent magnetic field fluctuations in both regions of space plasmas.

\section{Cluster and ACE data of 1 to 3 February 2002}

Figure 1 depicts the orbit trace of Cluster and spacecraft position of ACE, in the GSE Cartesian coordinate system, from 19:40:40 UT on 1 February 2002 to $03: 56: 38$ UT on 3 February 2002 during which Cluster-1 traverses the upstream region of the Earth's bow shock. For this time interval, ACE appears practically stationary in the scales of Fig. 1 and the Cluster tetrahedron scale was small ( 100-300 km). For spacecraft separations of $300 \mathrm{~km}$ and mean solar wind bulk velocity of $374 \mathrm{~km} / \mathrm{s}$ (obtained for the selected time interval) and assuming the Taylor's hypothesis, the time scale above which all 4 Cluster spacecraft observe the same ed- dies is $\sim 0.8 \mathrm{~s}$. In this paper, our analysis will cover time scales above $1 \mathrm{~s}$ (Fig. 10), hence the differences of measurements between the satellites are indistinguishable. The selected time interval, defined by the onset of the solar wind supersonic/subsonic transitions, begins when Cluster- 1 crosses the shock front of a quasi-perpendicular bow shock by entering into the solar wind at the time indicated by a dashed line in the upper panel of Fig. 2, and ends when Cluster-1 departs from the solar wind by entering into the transition (foreshock) region of a quasi-parallel bow shock at the time indicated by a dashed line in the lower panel of Fig. 2. In contrast to a quasi-perpendicular shock (Bale et al., 2005a) characterized by sharp transitions of the modulus of the ion bulk flow velocity $\left|V_{i}\right|$ and magnetic field $|B|$, a quasi-parallel shock (Burgess et al., 2005) is characterized by a transition region with repeated shock crossings, as seen in Fig. 2. This quasiparallel shock event has been analyzed by a number of papers (Eastwood et al., 2003; Stasiewicz et al., 2003; Behlke et al., 2004; Lucek et al., 2004). As mentioned in the Introduction, when the Cluster spacecraft navigate in the upstream solar wind they stay always very close to the bow shock, as a result magnetic connections to the bow shock occur frequently (Kellogg and Horbury, 2005). Although we have selected an interval outside of the foreshock region of a quasi-parallel bow shock the magnetic connection happens from time to time, for example, between 00:50 and 01:00 UT, and between 01:20 and 01:36 UT on 3 February 2002. Hence, the plasma conditions of solar wind seen by Cluster- 1 are different from 

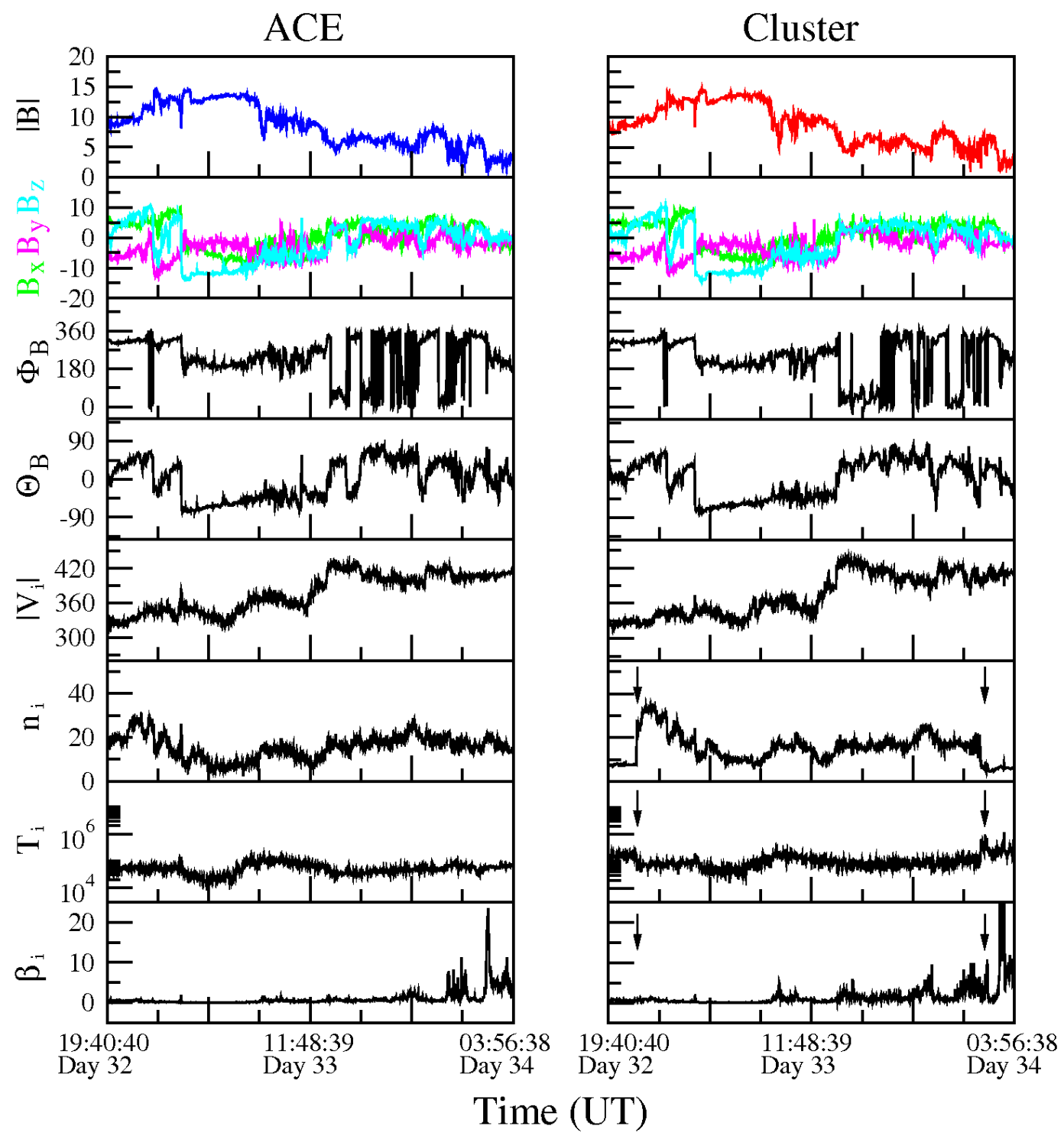

Fig. 3. ACE and Cluster-1 magnetic field and plasma parameters for the selected time interval. From top to bottom: modulus of magnetic field $|B|(\mathrm{nT})$; the three components of the vector magnetic field (nT) $B_{x}, B_{y}$, and $B_{z}$ in the GSE coordinates; angle $\Phi_{B}$ (degrees) of the magnetic field relative to the $\mathrm{x}$-axis in the ecliptic plane; angle $\Theta_{B}$ (degrees) of the magnetic field out of the ecliptic; ion bulk flow velocity $\left|V_{i}\right|(\mathrm{km} / \mathrm{s})$, ion number density $n_{i}\left(\mathrm{~cm}^{-3}\right)$, ion temperature $T_{i}$ (Kelvin), and ion plasma beta $\beta_{i}$.

that seen by ACE at L1 since the solar wind turbulence measured by Cluster- 1 is a combination of the ambient solar wind plus fluctuations coming from the bow shock.

In this paper, we perform a nonlinear analysis of the modulus of magnetic field $|B|=\left(B_{x}^{2}+B_{y}^{2}+B_{z}^{2}\right)^{1 / 2}$. We are interested in analyzing the relation between phase synchronization and intermittency of solar wind magnetic field turbulence which does not require a detailed analysis of its field components. As a matter of fact, in a similar study Bruno et al. (2003) showed that the modulus and the components of the solar wind magnetic field give the same qualitative behaviors of intermittency. The Cluster and ACE magnetic fields are detected by the FGM instruments (Balogh et al., 2001; Smith et al., 1998) at a resolution of $22 \mathrm{~Hz}$ and $1 \mathrm{~Hz}$, respectively, providing a set of 2604208 and 116159 data points, respectively, for the interval chosen. For the sake of completeness, Fig. 3 presents an overview of other in situ plasma parameters for this interval. The three components of the vector magnetic field $B_{x}, B_{y}$ and $B_{z}$ are given in the GSE coordinates. $\Phi_{B}$ and $\Theta_{B}$ denote the angle of the solar wind magnetic field relative to the Sun-Earth $\mathrm{x}$-axis in the ecliptic plane, and the angle out of the ecliptic, respectively, in the polar GSE coordinates (Eastwood et al., 2003). These angles can be obtained from the following relations 


$$
\begin{aligned}
& \Phi_{B}=\tan ^{-1}\left(\frac{B_{y}}{B_{x}}\right) \\
& \Theta_{B}=\tan ^{-1}\left(\frac{B_{z}}{\sqrt{B_{x}^{2}+B_{y}^{2}}}\right) .
\end{aligned}
$$

Figure 3 also shows the modulus of the ion bulk flow velocity $\left|V_{i}\right|$, the ion number density $n_{i}$ and the ion temperature $T_{i}$ (where the component perpendicular to the magnetic field for Cluster is plotted). It follows from Fig. 3 that during this time interval Cluster and ACE are immersed in a slow solar wind. The ion plasma beta $\beta_{i}$ (the ratio between plasma kinetic pressure and magnetic pressure) is calculated by the following expression

$\beta_{i}=\frac{2 \mu_{0} k_{B} n_{i} T_{i}}{B_{0}^{2}}$,

where $\mu_{0}=4 \pi \times 10^{-7}[\mathrm{Henry} / \mathrm{m}]$ is the permeability of vacuum, $k_{B}=1.38 \times 10^{-23}$ [Joule/K] is the Boltzmann constant, $n_{i}$ is the ion number density, $T_{i}$ is the ion temperature, and $B_{0}$ is defined here as the mean value of $|B|$. Plasma measurements from ACE are provided by the Solar Wind Electron Proton Alpha Monitor (McComas et al., 1998), while Cluster plasma measurements are given by the Ion Spectrometry experiment CIS (Rème et al., 2001). Note that the CIS instrument of Cluster-1 is switched from the telemetry mode 14 (Compression Magnetosphere-4) to the telemetry mode 5 (Compression Solar Wind-4) at 21:55:11 UT on Julian Day 32, and then to the telemetry mode 10 (Magnetosphere-3) at 01:15:04 UT on Julian Day 34 (Rème et al., 2001), which account for the discontinuities seen in the beginning and at the end of the ion number density and ion temperature profiles indicated by arrows in Fig. 3 .

Although the interplanetary magnetic field behaves sometimes as a stationary process (Bruno and Carbone, 2005), in the time interval studied here there is a trend in the time series of $|B|$ of Cluster and ACE, as seen in Fig. 3. In order to guarantee the stationarity of data we remove a trend from $|B|$ by subtracting a cubic fitting (Macek et al., 2005) computed from the time series of $|B|$. Figure 4 shows the resulting stationary time series of $|B|$ for Cluster and ACE, which display sporadic bursts of large-amplitude spikes typical of intermittency.

The upper panels of Fig. 5 show the power spectral density (PSD) of $|B|$ for Cluster and ACE, corresponding to the time series of $|B|$ of Fig. 4; they depict a typical power spectrum density of solar wind turbulence with a spectral break separating the inertial subrange from the dissipative subrange, each with its own power law (Leamon et al., 2000; Bruno et al., 2005; Alexandrova et al., 2008). The power spectral density was computed using the Welch method (Welch, 1967), which consists of dividing the time series into $M$ subintervals, multiplying each subinterval term by term by a
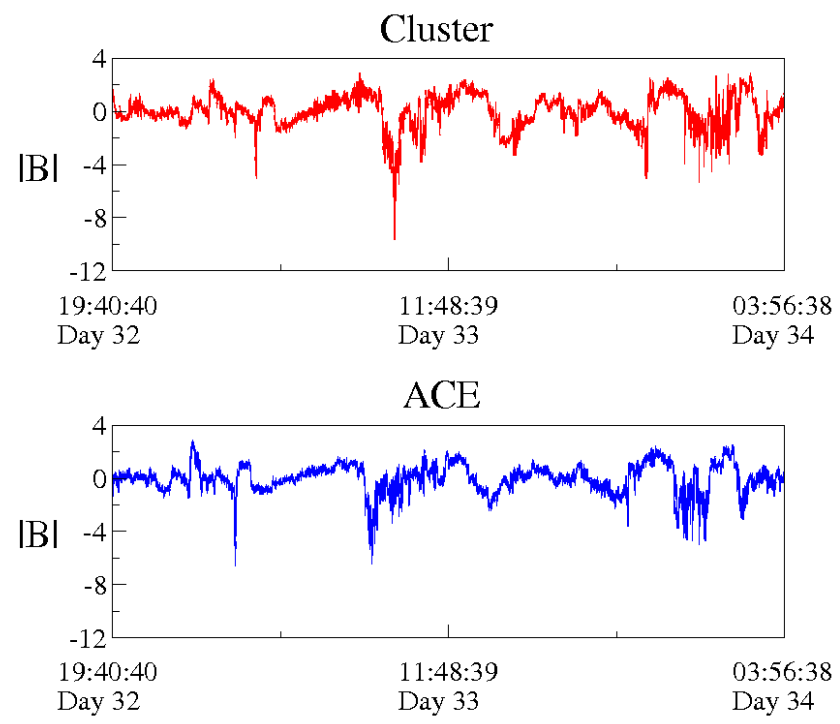

Fig. 4. Time series of the modulus of magnetic field $|B|$ (nT) of Cluster- 1 and ACE, after removing the trend by computing a cubic fitting of the original data.

window function (in our case we used the Hanning window (Paschmann and Daly, 2000)) and then computing the power spectrum of each subinterval using the fast Fourier transform. The average of the $M$ power spectra gives the PSD. This method reduces the error of the spectrum estimate, resulting in a narrower PSD. The spin frequency $f_{\text {spin }}$ of both spacecraft are indicated in Fig. 5. The Nyquist frequency $f_{\mathrm{Nyq}}$, defined as half of the sampling frequency $f_{s}, f_{\mathrm{Nyq}}=0.5 f_{s}$, which marks the maximum frequency for which the PSD gives reliable values (Paschmann and Daly, 2000), is equal to $11 \mathrm{~Hz}$ for Cluster and $0.5 \mathrm{~Hz}$ for ACE.

The frequency range in which each PSD follows a $-5 / 3$ Kolmogorov scaling (i.e., the inertial subrange) can be determined by constructing the compensated PSD, multiplying the original PSD by $f^{+5 / 3}$ (Biskamp et al., 1999). The inertial subrange should appear as a frequency range in which the compensated PSD is almost horizontal (i.e., zero slope). The compensated PSD of Cluster and ACE are shown in the lower panels of Fig. 5. To facilitate visualization, each compensated spectrum is smoothed by dividing it into overlapping subintervals shifted by one datapoint, each subinterval contains 10 datapoints, and then calculating the mean value within each subinterval. Each mean value is plotted at the center of the subinterval. The smoothed compensated spectra of Cluster and ACE are shown in the lower panels of Fig. 5 (black curves), and the vertical dashed lines indicate the beginning and the end of the resulting inertial subrange. For both Cluster and ACE, the beginning of the inertial subrange is defined as the first value of the smoothed compensated PSD. For Cluster, the compensated spectrum clearly shows a "knee" (i.e., a local maximum) to the right of $f_{\text {spin }}$, hence the end of the inertial subrange is defined as the 

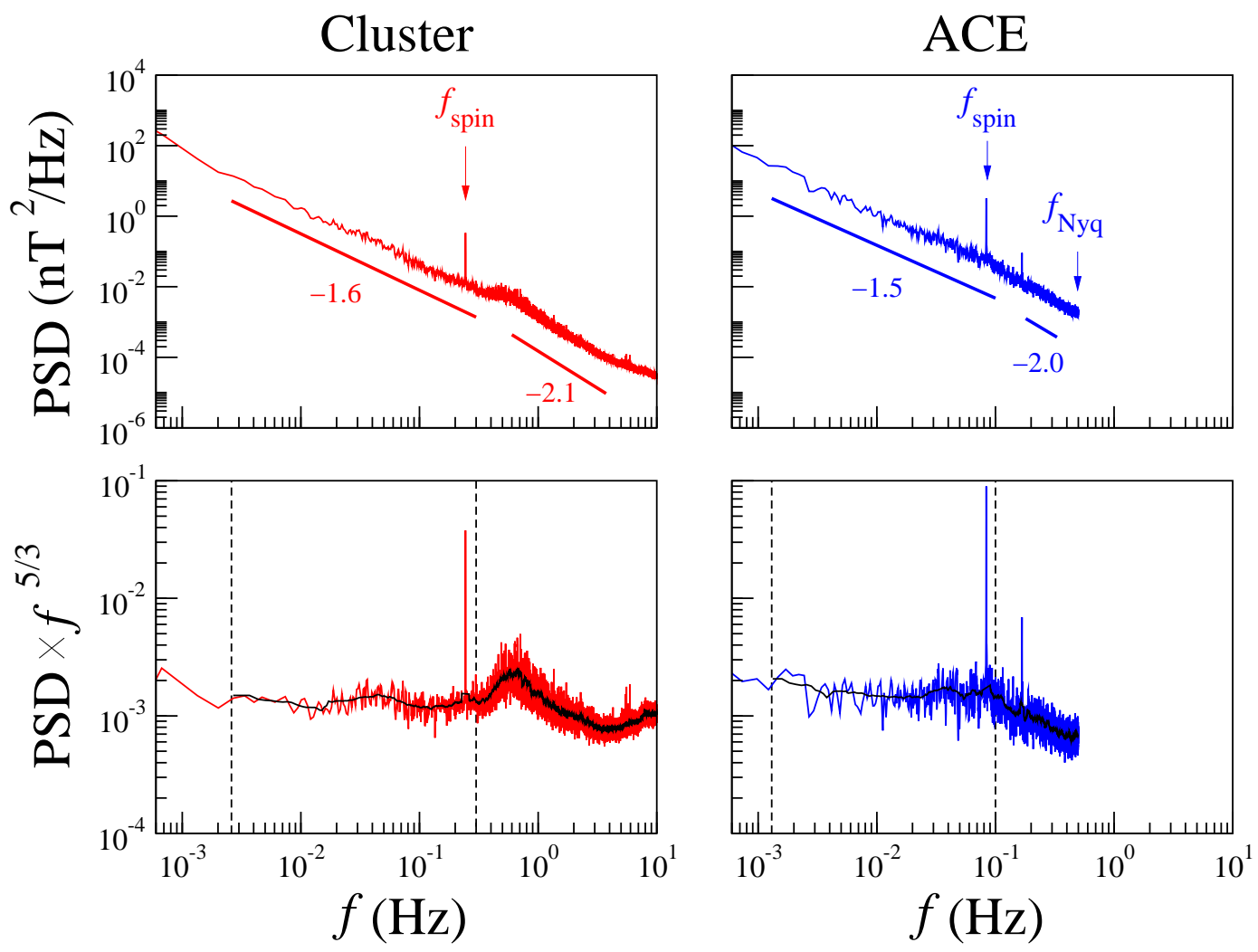

Fig. 5. Upper panels: Power spectral density (PSD) of $|B|$ for Cluster-1 and ACE. The spin frequency of each spacecraft is indicated as $f_{\text {spin }}$. The Nyquist frequency for ACE is indicated as $f_{\mathrm{Nyq}}$. Straight lines indicate the inertial and "dissipative" subranges of each spacecraft. The spectral break that marks the transition from the inertial to dissipative subrange occurs near the local ion cyclotron frequency $f_{c i}$. For both Cluster and ACE $f_{c i} \sim 0.12 \mathrm{~Hz}$ in the solar wind frame, which is Doppler-shifted to a higher frequency in the spacecraft frame. Lower panels: Compensated PSD for Cluster and ACE. To facilitate visualization, a smoothed compensated PSD is shown in black. Vertical dashed lines indicate the beginning and the end of the inertial subranges used to compute the spectral indices of Cluster and ACE in the upper panels.

frequency just before the "knee". For ACE, the end of the inertial subrange is defined as the frequency where a change of slope occurs. For Cluster, the inertial subrange spectral index in the frequency range $0.0026-0.3 \mathrm{~Hz}$ is $q_{\text {inert }}=-1.6 \pm 0.01$ and the dissipative subrange spectral index in the frequency range $0.6-3.74 \mathrm{~Hz}$ is $q_{\text {dissip }}=-2.1 \pm 0.01$. For ACE, the inertial subrange spectral index in the frequency range $0.0013-0.1 \mathrm{~Hz}$ is $q_{\text {inert }}=-1.5 \pm 0.01$ and the dissipative subrange spectral index in the frequency range $0.18-0.33 \mathrm{~Hz}$ is $q_{\text {dissip }}=-2.0 \pm 0.04$.

The fluctuations of the modulus of magnetic field $|B|$ can be regarded as compressible (or parallel) fluctuations (Samsonov et al., 2007; Alexandrova et al., 2008). The total power spectral density $\mathrm{PSD}_{\text {total }}$ is closer to the $-5 / 3$ Kolmogorov scaling than the PSD of $|B|\left(\mathrm{PSD}_{\|}\right)$, where $\mathrm{PSD}_{\text {total }}=\mathrm{PSD}\left(B_{x}\right)+\mathrm{PSD}\left(B_{y}\right)+\mathrm{PSD}\left(B_{z}\right)=\mathrm{PSD}_{\perp}+\mathrm{PSD}_{\|}$, $\mathrm{PSD}_{\perp}$ denotes the PSD of transverse Alfvénic fluctuations (Samsonov et al., 2007). In the solar wind at 1 AU, magnetic field fluctuations are mostly Alfvénic and nearly incompressible (Alexandrova, 2008) which implies that, within the inertial subrange, transverse fluctuations contain more power than compressible fluctuations.
The spectral break that marks the transition from the inertial to dissipative subrange occurs near the local ion cyclotron frequency $f_{c i}=e B / m_{i}$ in an appropriate frame. For both Cluster and ACE $f_{c i} \sim 0.12 \mathrm{~Hz}$ if we use $B_{0}$ in the solar wind frame. Since data are taken in the spacecraft frame, $f_{c i}$ has to be Doppler-shifted to higher frequency by a quantity of the order of $V_{s w} / V_{A}$, where $V_{s w}$ is the solar wind speed and $V_{A}$ is the Alfvén speed, in the interpretation of the power spectra in Fig. 5.

\section{Intermittency, non-Gaussianity and phase synchro- nization}

Figure 6 shows the scale dependence of the normalized magnetic field-differences of Cluster and ACE

$\Delta B=\frac{\delta B-\langle\delta B\rangle}{\sigma_{B}}$,

for three different time scales $(\tau=10 \mathrm{~s}, 100 \mathrm{~s}$ and $1000 \mathrm{~s})$, where $\delta B=|B(t+\tau)|-|B(t)|$ denotes two-point differences of the modulus of magnetic field $|B|$ for a given time scale 


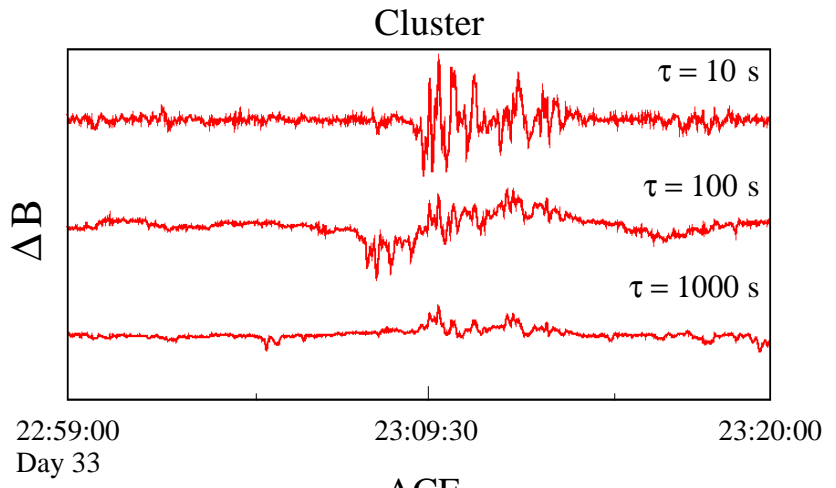

ACE

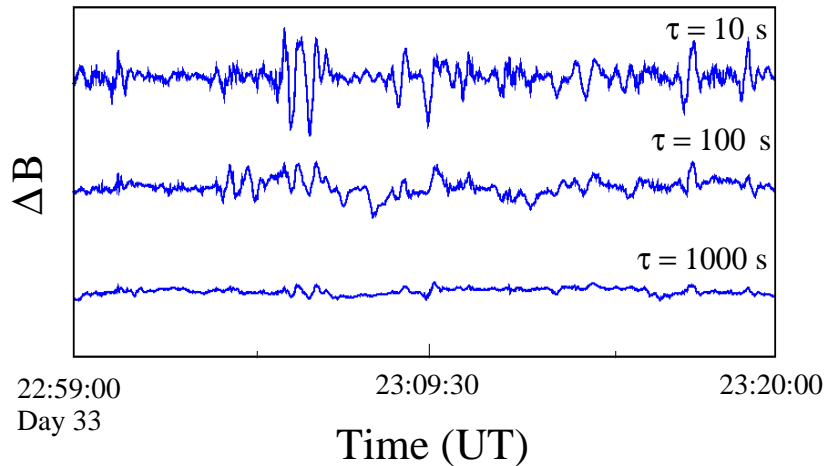

Fig. 6. Scale dependence of the normalized magnetic fielddifferences of Cluster-1 (red) and ACE (blue) for three different time scales ( $\tau=10 \mathrm{~s}, 100 \mathrm{~s}$ and $1000 \mathrm{~s})$.

(lag) $\tau$, the brackets denote the mean value of $\delta B$, and $\sigma_{B}$ denotes the standard deviation of $\delta B$. It is evident from Fig. 6 that, for both Cluster and ACE, the magnetic field fluctuations become more intermittent as the scales become smaller. In terms of spatial scales, the three time scales in Fig. 6 correspond to $3740 \mathrm{~km}, 37400 \mathrm{~km}, 374000 \mathrm{~km}$, respectively, using the mean solar wind (ion bulk flow) velocity of $374 \mathrm{~km} / \mathrm{s}$ and assuming the Taylor's hypothesis. Note that the spin frequency is filtered from the time series of Cluster and ACE, respectively, by applying an orthogonal wavelet decomposition to the data using a Daubechies 10 mother wavelet (Daubechies, 1994), and removing the scales corresponding to the spacecraft spintone of $0.25 \mathrm{~Hz}$ for Cluster and $0.083 \mathrm{~Hz}$ for ACE.

The intermittent characteristics of interplanetary turbulence can be elucidated by the probability density function (PDF) of magnetic field fluctuations. The $p$-th order of the structure function is formally defined as (de Wit, 2004)

$S_{p}(\tau)=\int_{-\infty}^{\infty} P(\Delta B(\tau))(\Delta B(\tau))^{p} d(\Delta B(\tau))$,

where $P(\Delta B)$ denotes the probability density function (PDF) of magnetic field differences $\Delta B$. The first four orders of the structure function are statistical quantities that characterize PDFs (Papoulis, 1965; Davidson, 2004), namely, $p=0$
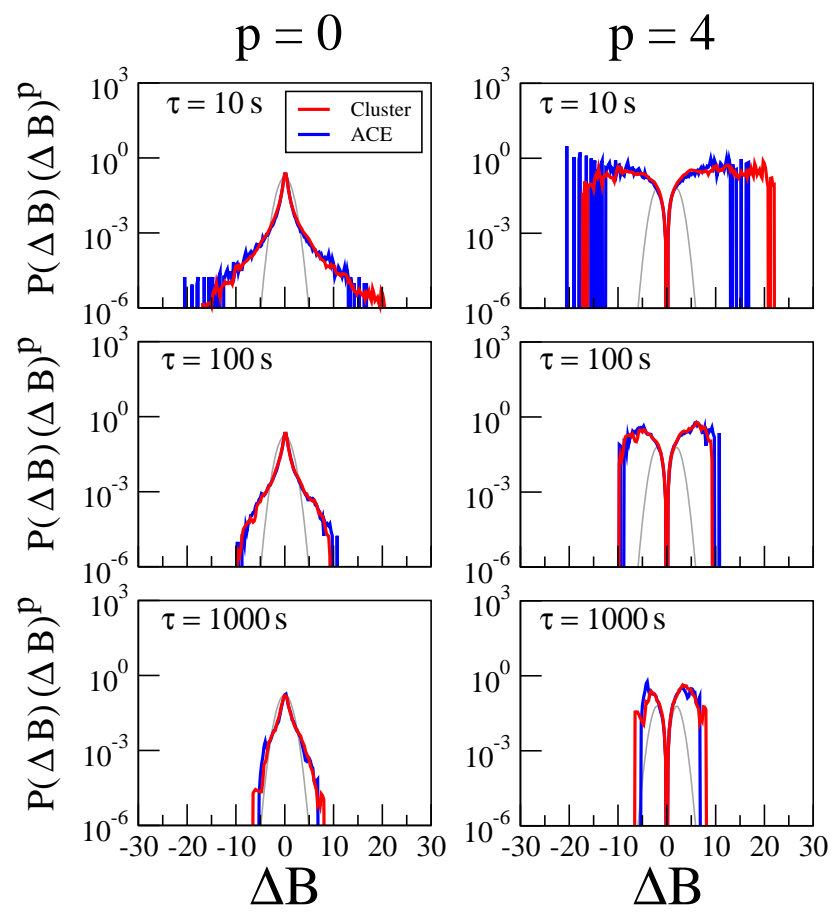

Fig. 7. The integrand of Eq. (2), for $p=0$ and $p=4$, determined from the magnetic field fluctuations of Cluster-1 (red) and ACE (blue), for three different time scales ( $\tau=10 \mathrm{~s}, 100 \mathrm{~s}$ and $1000 \mathrm{~s}$ ), superposed by a Gaussian PDF (grey line). The areas spanned by the curves of Cluster and ACE for $p=4$ approximate the value of the flatness.

gives the sum of all probabilities (equal to 1 by definition), $p=1$ gives the mean value of $\Delta B$ (equal to zero according to Eq. 1), $p=2$ gives the variance of $\Delta B$ (equal to 1 from Eq. 1 , whose square root is the standard deviation), $p=3$ measures the degree of asymmetry (skewness) of the distribution, and $p=4$ quantifies the flatness of the distribution. Figure 7 plots the integrand of Eq. (2) for $p=0$ (PDF) and 4 (flatness), determined from the magnetic field fluctuations of Cluster and ACE (Fig. 4), for 3 different time scales ( $\tau=10 \mathrm{~s}, 100 \mathrm{~s}$ and 1000 s), superposed by a Gaussian PDF (grey line). It shows that the PDFs of $\Delta B$ for both shocked and unshocked solar wind are closer to a Gaussian distribution at large time scales but deviate from a Gaussian distribution as $\tau$ decreases. At small scales the shape of PDF $(p=0)$ becomes leptokurtic, exhibiting fat tails and sharp peaks. For $p=4$, the flatness of the distribution of $\Delta B$ of both Cluster and ACE increases at small scales, indicating an excess of rare, large-amplitude fluctuations. The areas spanned by the curves shown in Fig. 7 for $p=4$ approximate the values of the fourth-order (flatness) structure function (de Wit, 2004). Table 1 lists numerical examples of flatness which shows that, for all three scales, the level of flatness of magnetic field fluctuations of ACE is higher than Cluster. 

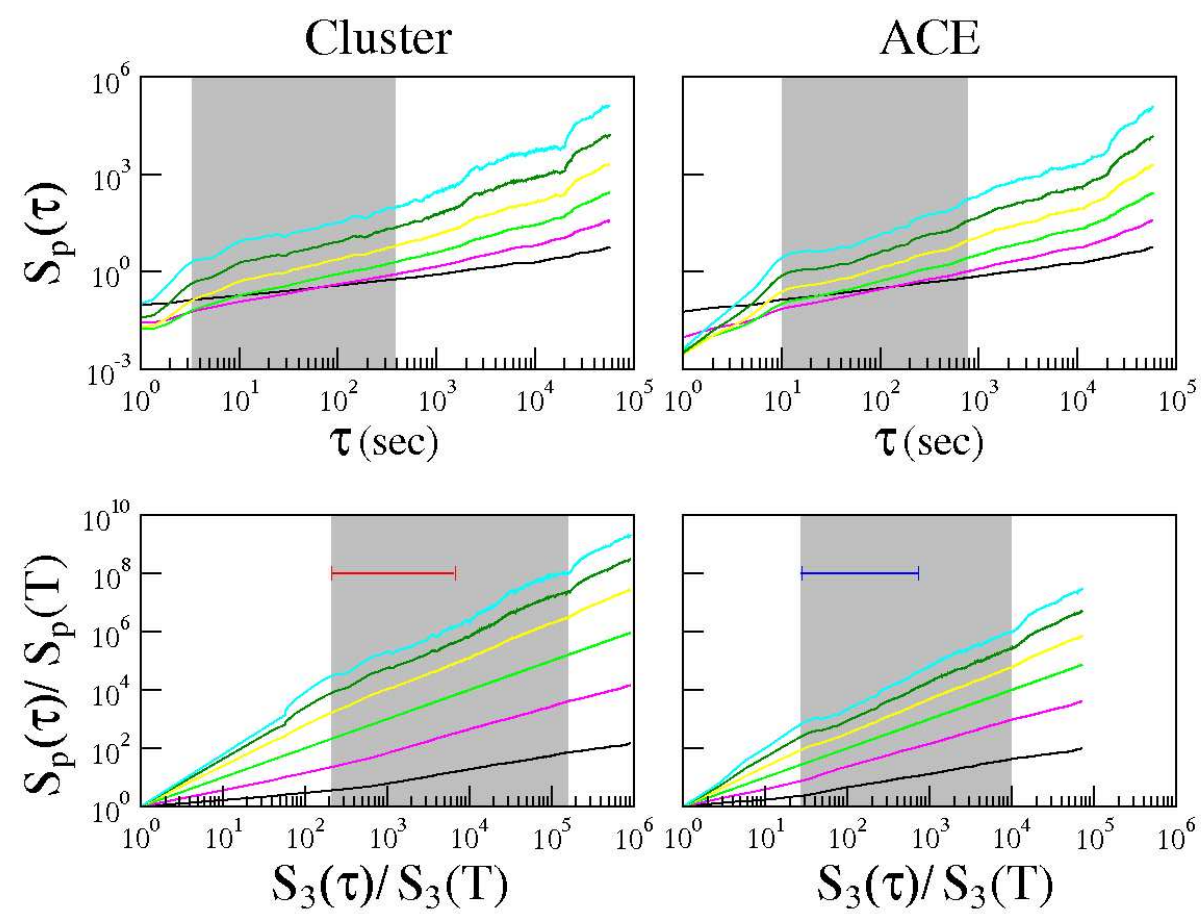

Fig. 8. Upper panels: variations of structure functions with timescale $\tau$ calculated from the magnetic field fluctuations of Cluster-1 and ACE (upper panels) for $p=1$ (black), 2 (purple), 3 (light green), 4 (yellow), 5 (dark green) and 6 (light blue), the grey area indicates the inertial subrange. Lower panels: structure functions after applying the Extended Self-Similarity technique, the bar indicates the previous inertial subrange, and the grey area indicates the extended scaling range. For the visualization purpose, the stretched structure functions have been normalized to $S_{p}(T)$, where $T=0.044 \mathrm{~s}$ for Cluster and $T=1 \mathrm{~s}$ for ACE.

Table 1. Numerical examples of flatness for three time scales.

\begin{tabular}{crrr}
\hline & $\tau=10 \mathrm{~s}$ & $100 \mathrm{~s}$ & $1000 \mathrm{~s}$ \\
\hline Cluster & 35.86 & 14.41 & 6.80 \\
ACE & 47.39 & 16.02 & 7.67 \\
\hline
\end{tabular}

The departure from self-similarity in the magnetic field fluctuations can be quantified by comparing the scaling exponents of higher-order structure functions within the inertial subrange against the Kolmogorov universality theory (Frisch, 1995). The characterization of departures from Kolmogorov's 1941 theory (hereafter K41) is of great interest since the K41 theory is a result based on the assumption that the turbulence is homogeneous and isotropic (i.e., selfsimilar). The upper panels of Fig. 8 show the structure functions obtained from the following formula (de Wit and Krasnoselskikh, 1996),

$S_{p}(\tau)=\left\langle\left|B_{i+\tau}-B_{i}\right|^{p}\right\rangle \sim \tau^{\alpha(p)}$,

for $p=1-6$. The scale is logarithmic for both axis. The grey areas denote the inertial subranges determined from the power spectral density of Fig. 5. The scaling exponent for each order of the structure function can be obtained by estimating the slope of a linear-fitting of the curves within the inertial subrange. In order to improve the calculation of the scaling exponent, we apply the Extended SelfSimilarity (ESS) technique (Benzi et al., 1993), which consists of plotting each order of the structure function $S_{p}$ as a function of $S_{3}$. This technique allows us to extend the scaling range where $S_{p}(\tau) \sim\left[S_{3}(\tau)\right]^{\zeta(p)}$. The scaling exponents $\zeta(p) \sim \alpha(p) / \alpha(3)$ can be found from the extended range. The lower panels of Fig. 8 illustrate the application of this technique. The horizontal line represents the inertial subrange before the "stretching" process, and the grey area indicates the extended scaling range. Figure 9 shows the scaling exponent $\zeta(p)$ as a function of $p$, for Cluster and ACE. The dashed line denotes the $\mathrm{K} 41$ scaling, $\zeta(p)=p / 3$. It is evident, from Fig. 9, that the scaling exponent measured by both spacecraft display significant departure from self-similarity, which implies that the magnetic field fluctuations in both regions of space plasmas are intermittent. For the time interval considered in this paper, the unshocked solar wind magnetic field at L1 is more intermittent than the shocked solar wind upstream of Earth's bow shock, in agreement with Table 1.

Intermittency can be quantified by calculating the empirical estimate of the normalized fourth-order moment $K$ (kurtosis) (de Wit, 2004),

$K(\tau)=\frac{1}{n} \sum_{i=1}^{n}\left(\frac{\delta B_{i}-\left\langle\delta B_{i}\right\rangle}{\sigma_{B}}\right)^{4}-3$, 


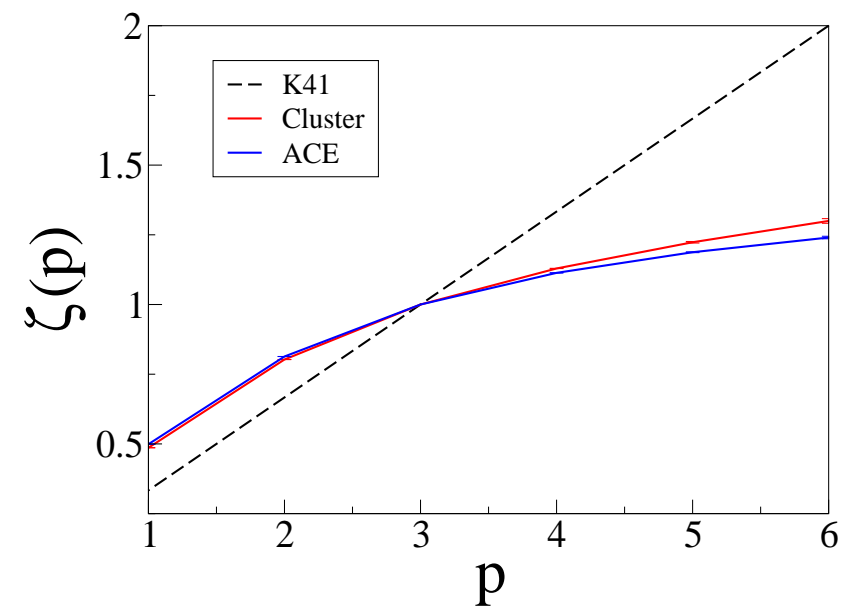

Fig. 9. Scaling exponent $\zeta$ of the $p$-th order structure function obtained by ESS fitting for Cluster-1 and ACE magnetic field fluctuations. The dashed line corresponds to K41 (self-similar) Kolmogorov scaling.

which is equivalent to flatness minus 3 (Frisch, 1995; Davidson, 2004). For a Gaussian signal $K=0$ for all scales; whereas for an intermittent (non-Gaussian) signal $K(\tau)>0$ and $K$ increases as scale decreases within the inertial subrange. Figure 10 shows the computed variation of $K$ with the time scale $\tau$ for magnetic field fluctuations of Cluster and ACE. We have chosen the lower bound of $1 \mathrm{~s}$ for Fig. 10 since the measurements at scales smaller than $1 \mathrm{~s}$ are contaminated by the noise level of FGM instruments. The test to find the smallest time scale is done by over-plotting the instrument noise level of $10^{-4} \mathrm{nT}^{2} / \mathrm{Hz}$ (for both Cluster and ACE) on the power spectral density of Fig. 5 .

An alternative method of quantifying intermittency and non-Gaussianity is to apply the phase coherence technique using surrogate data by defining a phase coherence index (Hada et al., 2003; Koga and Hada, 2003; Koga et al., 2007, 2008; Nariyuki and Hada, 2006; Chian et al., 2008; Nariyuki et al., 2008; Sahraoui, 2008; Telloni et al., 2009)

$C_{\phi}(\tau)=\frac{S_{\mathrm{PRS}}(\tau)-S_{\mathrm{ORG}}(\tau)}{S_{\mathrm{PRS}}(\tau)-S_{\mathrm{PCS}}(\tau)}$,

where

$S_{j}(\tau)=\sum_{i=1}^{n}\left|B_{i+\tau}-B_{i}\right|$,

with $j=\mathrm{ORG}$, PRS, PCS. This index measures the degree of phase synchronization in an original data set (ORG) by comparing it with two surrogate data sets created from the original data set: a phase-randomized surrogate (PRS) in which the phases of the Fourier modes are made completely random, and a phase-correlated surrogate (PCS) in which the phases of the Fourier modes are made completely equal. The power spectrum of three data sets ORG, PRS and PCS are
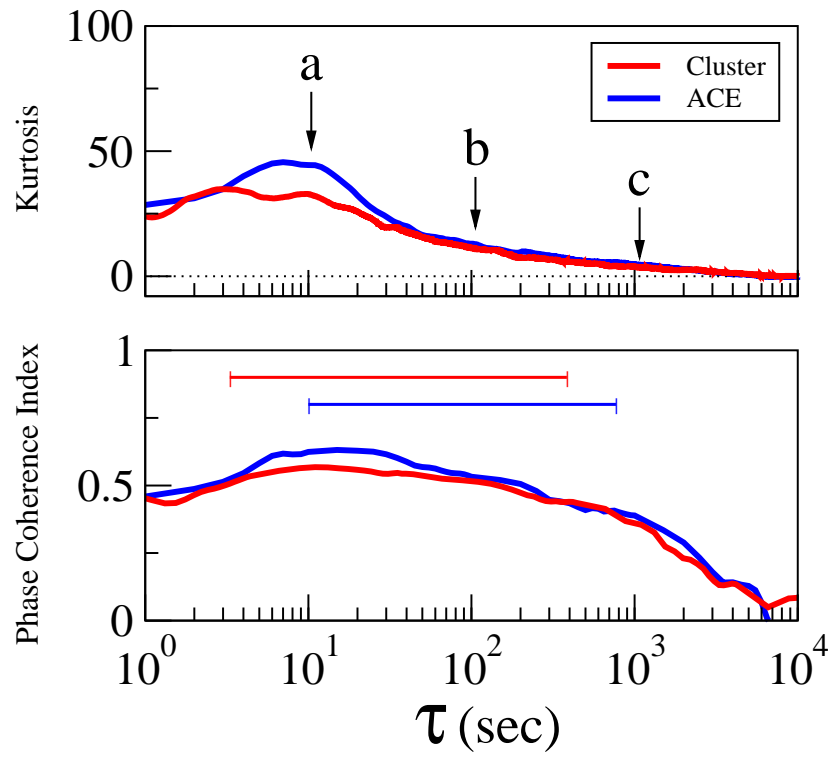

Fig. 10. Kurtosis and phase coherence index of $|B|$ measured by Cluster-1 (red) and ACE (blue). Letters a, b and c indicate scales $\tau=10,100$ and $1000 \mathrm{~s}$, respectively. The bars indicate the inertial subrange of each spacecraft obtained from Fig. 5. The inverse of the ion cyclotron frequency $f_{c i} \sim 0.12 \mathrm{~Hz}$ in the solar wind frame is $\tau \sim 8.3 \mathrm{~s}$, which is near the peak regions of kurtosis and phase coherence index.

kept the same, but their phase spectra are different. An average of over 100 realizations of the phase shuffling is performed to generate the phase-randomized surrogate data set $S_{\mathrm{PRS}}(\tau) . C_{\phi}(\tau)=0$ indicates that the phases of the scales $\tau$ of the original data are completely random (i.e., null phase synchronization), whereas $C_{\phi}(\tau)=1$ indicates that the phases are fully correlated (i.e., total phase synchronization). Figure 10 displays the computed variation of $C_{\phi}$ with the time scale $\tau$ for magnetic field fluctuations of Cluster and ACE, whose behaviors follow that of kurtosis.

The upper panel of Fig. 10 shows the variation of kurtosis as a function of the time scale $\tau$. For large scales $\left(\tau \gtrsim 10^{3} \mathrm{~s}\right)$ kurtosis is nearly zero, implying that the magnetic field fluctuations are near-Gaussian. For $10 \mathrm{~s} \lesssim \tau \lesssim 10^{3} \mathrm{~s}$, kurtosis increases as the time scale decreases which characterizes intermittency and non-Gaussianity related to nonlinear energy cascade within the inertial subrange seen in Fig. 5. The lower panel of Fig. 10 shows the variation of the phase coherence index with $\tau$ which presents similar characteristics of kurtosis, indicating that phase synchronization due to nonlinear multi-scale interactions is responsible for intermittency. The inertial subranges for Cluster and ACE (obtained from Fig. 5) are marked with a bar in Fig. 10. The results of Fig. 10 display similar trend as the upstream results of Koga et al. (2007) obtained by the Geotail data at the Earth's bow shock in the sense that, as the scale $\tau$ decreases, both kurtosis and phase coherence index increase until a certain scale 
where they reach their respective maxima, and then both kurtosis and phase coherence index start to decrease as $\tau$ decreases.

We conclude from Fig. 10 that either kurtosis or phase coherence index can be used to determine the degree of intermittency and phase synchronization in solar wind turbulence. Both nonlinear techniques prove that the solar wind magnetic field fluctuations, measured by Cluster and ACE, are intermittent (non-Gaussian) exhibiting high degree of intermittency (non-Gaussianity) at small scales and low degree of intermittency (near-Gaussianity) at large scales, in complete agreement with Figs. 6-9. It is interesting to point out that the period of $10 \mathrm{~s}$ of Alfvén waves analyzed by Eastwood et al. (2003) in the same Cluster event, from 04:02:30 UT to 04:10:00 UT (outside of our interval), is close to the peak of Fig. 10 where the intermittency is strongest. In addition, solar wind turbulence can be decomposed into coherent (non-Gaussian) and incoherent (Gaussian) components using the local intermittency measure analysis (Alexandrova et al., 2008).

\section{Conclusion}

For numerical simulations and analytic formulation of turbulence based on a set of deterministic plasma or fluid equations, in the absence of noise, it is natural to expect that a departure from Gaussianity arises from nonlinear multiscale coupling in turbulent energy cascade (Frisch, 1995; Davidson, 2004). In contrast, the observational data of space plasma turbulence is an admixture of deterministic signal and stochastic noise. Under this circumstance, a demonstration of finite phase synchronization is required to ascertain the nonlinear origin of non-Gaussian fluctuations.

Figure 10 shows that, within the inertial subrange, the departure from Gaussianity increases as the scale decreases, which is a characteristic of intermittent turbulence. Moreover, Fig. 10 shows that phase synchronization associated with nonlinear multiscale interactions is the origin of intermittency and non-Gaussianity, which leads to the formation of large-amplitude phase coherent (intermittent) structures at small scales. We have identified these large-amplitude phase coherent structures as spikes in the time series of magneticfield differences in Fig. 6 and fat tails in the PDF and extended flatness in Fig. 7. Our computed results are consistent with the Helios analysis by Bruno et al. (2003) of solar wind intermittency in the inner heliosphere. For slow solar wind at $0.9 \mathrm{AU}$, Bruno et al. (2003) obtained the values of 19 for $\tau=100 \mathrm{~s}$ and 7 for $\tau=1000 \mathrm{~s}$ for flatness, which are close to our numerical examples given in Table 1.

Large-amplitude coherent structures embedded within intermittent magnetic field turbulence in the foreshock region of Earth's bow shock have been detected by Cluster. Eastwood et al. (2003) detected, on 3 February 2002, largeamplitude ultra-low-frequency (ULF) Alfvén waves with wavelength of $3400 \mathrm{~km}$, in the foreshock region of Earth's quasi-parallel bow shock. Stasiewicz et al. (2003) reported the measurement, on 3 February 2002, of the density profiles and wave spectra inside fast magnetosonic shocklets, $1000 \mathrm{~km}$ in size and amplitude of 10 times the ambient magnetic field, upstream of a quasi-parallel bow shock. Behlke et al. (2004) observed on 3 February 2002 solitary waves, as bipolar pulses in the spiky electric field moving at velocities of $400-1200 \mathrm{~km} / \mathrm{s}$ along the ambient magnetic field with peak-to-peak amplitudes of $E_{\|}=65 \mathrm{mV} / \mathrm{m}$ and parallel scale sizes of $L_{\|} \sim 300-600 \mathrm{~m} \sim 10 \lambda_{D}$ (Debye length), within short large-amplitude magnetic structures (SLAMS) upstream of a quasi-parallel bow shock. In addition, Parks et al. (2006) detected ion density holes accompanied by magnetic holes $(\sim 3700 \mathrm{~km})$ upstream of a quasi-parallel bow shock, which are seen only with upstream particles, suggesting a link with backstreaming particles interacting with the solar wind. All the aforementioned observations (found outside of the time interval analyzed in this paper) refer to the foreshock region of a quasi-parallel shock which is a patchwork of SLAMS slowing down and piling up. SLAMS evolve from ULF instabilities excited by counterstreaming plasma populations (Schwartz, 2006) and have amplitudes 2-4 times larger than the ambient magnetic field, with typical durations of around $10 \mathrm{~s}$ and transverse dimensions of $\sim 1 R_{E}$ (Lucek et al., 2004). Although the interval of Cluster data analyzed in this paper is outside of the foreshock region, we expect that some of the coherent structures discussed above may contribute to the intermittent turbulence in this upstream region when IMF connects to bow shock (Kellogg and Horbury, 2005).

Large-amplitude coherent structures in the ambient solar wind have been found by Helios and ACE. Bruno et al. $(2001,2003,2005)$ reported the observation of coherent structures in the intermittent magnetic field turbulence between 0.3 to $1 \mathrm{AU}$ using the Helios solar wind data. During a fast solar wind interval on Julian Day 49-52 1976 when Helios-2 was at 0.9 AU and the solar wind fluctuations are Alfvénic, they detected one coherent structure related to a flux tube of scale size $\sim 9 \times 10^{5} \mathrm{~km}$ (Bruno et al., 2001). In a recent paper, Borovsky (2008) studied the statistics of 65,860 flux tubes in the ACE data for the period 1998-2004, and obtained a median scale size of flux tubes of $\sim 6.2 \times 10^{5} \mathrm{~km}$ for slow solar wind and of $\sim 4.2 \times 10^{5} \mathrm{~km}$ for fast solar wind. These coherent structures (flux tubes) are spotted by large changes in the magnetic field direction and the vector flow velocity, and are associated with large changes in the ion entropy density and the alpha-to-proton ratio. These flux tubes map to granule and supergranule sizes on the Sun's photosphere. Borovsky (2008) suggested a method for using these solar wind coherent structures for remote sensing of the dynamics of the Sun's magnetic carpet. Note that the coherent structures reported by Bruno et al. $(2001,2003,2005)$ and Borovsky (2008) have spatial scales $\sim 10^{5} \mathrm{~km}$ which correspond to time scales $\sim 10^{3} \mathrm{~s}$ (assuming Taylor's hypothesis 
and $V_{s w} \sim 4 \times 10^{2} \mathrm{~km} / \mathrm{s}$ ). These large-scale flux tubes might be coherent structures convected by the solar wind from the base of the solar atmosphere (Bruno et al., 2001; Borovsky, 2008).

It is worth mentioning that the most intermittent events in the solar wind turbulence, which occur at time scales of the order of a few minutes, have been identified as current sheets and shock waves (Veltri and Mangeney, 1999; Veltri, 1999; Alexandrova, 2008). These small-scale intermittent structures may be related to locally occurring magnetic reconnections (Chang et al., 2004; Zhou et al., 2004; Greco et al., 2009) and may play an important role in the phase synchronization observed by Fig. 10 in the interplanetary magnetic field turbulence.

The results of this paper provide the first observational proof of phase coherence in the ambient solar wind turbulence, based on the magnetic field data of ACE at L1. Figure 10 indicates that the level of intermittency and phase synchronization detected by both Cluster and ACE are very similar except for scales around $10 \mathrm{~s}$, where ACE observed a higher level of intermittency and phase synchronization than Cluster. The peak regions of kurtosis and phase coherence index in Fig. 10 corresponds to the spectral break regions in Fig. 5 where the magnetic field turbulence is dominated by nonlinear wave-wave and wave-particle interactions. Since Cluster is located in the shocked solar wind, the reflected ions from the Earth's bow shock can enhance the dissipation of nonlinear Alfvén waves via ion-cyclotron damping and other kinetic effects (Howes et al., 2008), leading to a decrease of phase synchronization.

In conclusion, our study based on Cluster and ACE observations demonstrate that the intermittency in the magnetic field turbulence, in the shocked solar wind upstream of Earth's bow shock and in the unshocked ambient solar wind at $\mathrm{L} 1$, is the result of phase synchronization intrinsic in nonlinear multiscale interactions. Numerical simulations of nonlinear plasma waves have confirmed that intermittent events are localized regions of plasmas (or fluids) governed by bursts of energy spikes (phase coherent structures) where phase synchronization is operating (He and Chian, 2003, 2005). Since large-amplitude coherent structures of small scales have typical lifetimes longer than that of small-amplitude incoherent (stochastic) fluctuations, the dynamics of an intermittent turbulence, ubiquitous in the heliophysical environment (Chian et al., 2006), is dominated by coherent structures resulting from phase synchronization.

Acknowledgements. The authors wish to thank O. Alexandrova, M. L. Goldstein and K. Stasiewicz for stimulating discussions. Valuable comments of the referees are greatly appreciated. We thank the Cluster FGM and CIS instrument teams, and the ACE MAG and SWEPAM instrument teams for providing the data. This research is supported by CNPq and FAPESP.

Topical Editor R. Nakamura thanks two anonymous referees for their help in evaluating this paper.

\section{References}

Alexandrova, O., Carbone, V., Veltri, P., and Sorriso-Valvo, L.: Solar wind Cluster observations: Turbulent spectrum and role of Hall effect, Planet. Space Sci., 55, 2224-2227, doi:10.1016/j.pss.2007.05.022, 2007.

Alexandrova, O., Carbone, V., Veltri, P., and Sorriso-Valvo, L.: Small-scale energy cascade of the solar wind turbulence, Astrophys. J., 674, 1153-1157, 2008.

Bale, S. D., Balikhin, M. A., Horbury, T. S., Krasnoselskikh, V. V., Kucharek, H., Möbius, E., Walker, S. N., Balogh, A., Burgess, D., Lembège, B., Lucek, E. A., Scholer, M., Schwartz, S. J., and Thomsen, M. F.: Quasi-perpendicular shock structure and processes, Space Sci. Rev., 118, 161-203, doi:10.1007/s11214005-3827-0, 2005a.

Bale, S. D., Kellogg, P. J., Mozer, F. S., Horbury, T. S., and Rème, H.: Measurement of the electric fluctuation spectrum of magnetohydrodynamic turbulence, Phys. Rev. Lett., 94, 215002, doi:10.1103/PhysRevLett.94.215002, 2005b.

Balogh, A., Carr, C. M., Acua, M. H., Dunlop, M. W., Beek, T. J., Brown, P., Fornacon, K.-H., Georgescu, E., Glassmeier, K.H., Harris, J., Musmann, G., Oddy, T., and Schwingenschuh, K.: The Cluster Magnetic Field Investigation: overview of in-flight performance and initial results, Ann. Geophys., 19, 1207-1217, 2001, http://www.ann-geophys.net/19/1207/2001/.

Behlke, R., André, M., Bale, S. D., Pickett, J. S., Cattell, C. A., Lucek, E. A., and Balogh, A.: Solitary structures associated with short large-amplitude magnetic structures (SLAMS) upstream of the Earth's quasi-parallel bow shock, Geophys. Res. Lett., 31, L16805, doi:10.1029/2004GL019524, 2004.

Benzi, R., Ciliberto, S., Tripiccione, R., Baudet, C., Massaioli, F., and Succi, S.: Extended self-similarity in turbulent flows, Phys. Rev. E, 48, R29-R32, 1993.

Biskamp, D., Schwarz, E., Zeiler, A., Celani, A., and Drake, J. F.: Electron magnetohydrodynamic turbulence, Phys. Plasmas, 6(3), 751-758, 1999.

Borovsky, J. E.: Flux tube texture of the solar wind: Strands of the magnetic carpet at 1 AU?, J. Geophys. Res.-Space Phys., 113, A08110, doi:10.1029/2007JA012684, 2008.

Bruno, R. and Carbone, V.: The solar wind as a turbulence laboratory, Living Rev. Solar Phys., 2, 4, 2005.

Bruno, R., Carbone, V., Veltri, P., Pietropaolo, E., and Bavassano, B.: Identifying intermittency events in the solar wind, Planet. Space Sci., 49, 1201-1210, 2001.

Bruno, R., Carbone, V., Sorriso-Valvo, L., and Bavassano, B.: Radial evolution of solar wind intermittency in the inner heliosphere, J. Geophys. Res.-Space Phys., 108(A3), 1130, doi:10.1029/2002JA009615, 2003.

Bruno, R., Carbone, V., Bavassano, B., and Sorriso-Valvo, L.: Observations of magnetohydrodynamic turbulence in the 3D heliosphere, Adv. Space Res., 35, 939-950, doi:10.1016/j.asr.2005.01.106, 2005.

Burgess, D., Lucek, E. A., Scholer, M., Bale, S. D., Balikhin, M. A., Balogh, A., Horbury, T. S., Krasnoselskikh, V. V., Kucharek, H., Lembège, B., Möbius, E., Schwartz, S. J., Thomsen, M. F., and Walker, S. N.: Quasi-parallel shock structure and processes, Space Sci. Rev., 118, 205-222, doi:10.1007/s11214-005-3832-3, 2005.

Burlaga, L. F. and Viñas, A. F.: Multi-scale probability distributions of solar wind speed fluctuations at 1 AU described by a general- 
ized Tsallis distribution, Geophys. Res. Lett., 31(16), L16807, doi:10.1029/2004GL020715, 2004.

Chang, T., Tam, S., and Wu, C.-C.: Complexity induced anisotropic bimodal intermittent turbulence in space plasmas, Phys. Plasmas, 11(4), 1287-1299, doi:10.1063/1.1667496, 2004.

Chapman, S. C. and Hnat, B.: Quantifying scaling in the velocity field of the anisotropic turbulent solar wind, Geophys. Res. Lett., 34(17), L17103, doi:10.1029/2007GL030518, 2007.

Chian, A. C.-L., Kamide, Y., Rempel, E. L., and Santana, W. M.: On the chaotic nature of solar-terrestrial environment: Interplanetary Alfvén intermittency, J. Geophys. Res., 111, A07S03, doi:10.1029/2005JA011396, 2006.

Chian, A. C.-L., Miranda, R. A., Koga, D., Bolzan, M. J. A., Ramos, F. M., and Rempel, E. L.: Analysis of phase coherence in fully developed atmospheric turbulence: Amazon forest canopy, Nonlinear Proc. Geophys., 15, 567-573, 2008.

Davidson, P. A.: Turbulence: an introduction for scientists and engineers, Oxford University Press, New York, 2004.

Daubechies, I.: Ten lectures on wavelets, CBMS, SIAM, 1994.

de Wit, T. D.: Can high-order moments be meaningfully estimated from experimental turbulence measurements?, Phys. Rev. E, 70, 055302, doi:10.1103/PhysRevE.70.055302, 2004.

de Wit, T. D. and Krasnosel'skikh, V. V.: Non-Gaussian statistics in space plasma turbulence: fractal properties and pitfalls, Nonlin. Processes Geophys., 3, 262-273, 1996, http://www.nonlin-processes-geophys.net/3/262/1996/.

Eastwood, J. P., Balogh, A., Lucek, E. A., Mazelle, C., and Dandouras, I.: On the existence of Alfvén waves in the terrestrial foreshock, Ann. Geophys., 21, 1457-1465, 2003, http://www.ann-geophys.net/21/1457/2003/.

Frisch, U.: Turbulence - The Legacy of A. N. Kolmogorov, Cambridge Univ. Press, Cambridge, 1995.

Greco, A., Matthaeus, W. H., Servidio, S., Chuychai, P., and Dmitruk, P.: Statistical analysis of discontinuities in solar wind ACE data and comparison with intermittent MHD turbulence, Astrophys. J. Lett., 691, L111-L114, doi:10.1088/0004637X/691/2/L111, 2009.

Hada, T., Koga, D., and Yamamoto, E.: Phase coherence of MHD waves in the solar wind, Space Sci. Rev., 107, 463-466, doi:10.1023/A:1025506124402, 2003.

Hamilton, K., Smith, C. W., Vasquez, B. J., and Leamon, R. J.: Anisotropies and helicities in the solar wind inertial and dissipation ranges at $1 \mathrm{AU}$, J. Geophys. Res.-Space Phys., 113(A1), A01106, doi:10.1029/2007JA012559, 2008.

$\mathrm{He}, \mathrm{K}$. and Chian, A. C.-L.: On-off collective imperfect phase synchronization and bursts in wave energy in a turbulent state, Phys. Rev. Lett., 91, 3, 034102, doi:10.1103/PhysRevLett.91.034102, 2003.

He, K. and Chian, A. C.-L.: Nonlinear dynamics of turbulent waves in fluids and plasmas, Nonlin. Processes Geophys., 12, 13-24, 2005 , http://www.nonlin-processes-geophys.net/12/13/2005/.

Howes, G. G., Dorland, W., Cowley, S. C., Hammett, G. W., Quataert, E., Schekochihin, A. A., and Tatsuno, T.: Kinetic simulations of magnetized turbulence in astrophysical plasmas, Phys. Rev. Lett., 100, 065004, doi:10.1103/PhysRevLett.100.065004, 2008.

Kellogg, P. J. and Horbury, T. S.: Rapid density fluctuations in the solar wind, Ann. Geophys., 23, 3765-3773, 2005, http://www.ann-geophys.net/23/3765/2005/.

Koga, D. and Hada, T.: Phase coherence of foreshock MHD waves: wavelet analysis, Space Sci. Rev., 107, 495-498, doi:10.1023/A:1025510225311, 2003.

Koga, D., Chian, A. C.-L., Miranda, R. A., and Rempel, E. L.: Intermittent nature of solar wind turbulence near the Earth's bow shock: Phase coherence and non-Gaussianity, Phys. Rev. E, 75, 046401, doi:10.1103/PhysRevE.75.046401, 2007.

Koga, D., Chian, A. C.-L., Hada, T., and Rempel, E. L.: Experimental evidence of phase coherence of magnetohydrodynamic turbulence in the solar wind: GEOTAIL satellite data, Phil. Trans. Royal Soc. London - Math. and Phys. Sci., 366, 1864, 447-457, doi:10.1098/rsta.2007.2102, 2008.

Leamon, R. J., Matthaeus, W. H., Smith, C. W., Zank, G. P., Mullan, D. J., and Oughton, S.: MHD-driven kinetic dissipation in the solar wind and corona, Astrophys. J., 537, 1054-1062, 2000.

Lucek, E. A., Horbury, T. S., Balogh, A., Dandouras, I., and Rème, H.: Cluster observations of structures at quasi-parallel bow shocks, Ann. Geophys., 22, 2309-2313, 2004,

http://www.ann-geophys.net/22/2309/2004/.

Macek, W. M., Bruno, R., and Consolini, G.: Generalized dimensions for fluctuations in the solar wind, Phys. Rev. E, 72, 017202, doi:10.1103/PhysRevE.72.017202, 2005.

McComas, D. J., Bame, S. J., Barker, P., Feldman, W. C., Phillips, J. L., Riley, P., and Griffee, J. W. : Solar Wind Electron Proton Alpha Monitor (SWEPAM) for the Advanced Composition Explorer, Space Sci. Rev., 86, 563-612, 1998.

Narita, Y., Glassmeier, K.-H., and Treumann, R. A.: Wave-number spectra and intermittency in the terrestrial foreshock region, Phys. Rev. Lett., 97, 191101, doi:10.1103/PhysRevLett.97.191101, 2006.

Nariyuki, Y. and Hada, T.: Remarks on nonlinear relation among phases and frequencies in modulational instabilities of parallel propagating Alfvén waves, Nonlin. Processes Geophys., 13, 425-441, 2006,

http://www.nonlin-processes-geophys.net/13/425/2006/.

Nariyuki, Y., Hada, T., and Tsubouchi, K.: On nonlinear evolution of Alfvénic turbulence in low beta plasmas, Phys. Plasmas 15, 114502, doi:10.1063/1.3028316, 2008.

Pallocchia, G., Bruno, R., Bavassano, B., et al.: Turbulence in the solar wind as seen by Cluster CIS experiment: Preliminary results on intermittency and scaling laws, SOLSPA 2001, Proceedings of the Second Solar Cycle and Space Weather Euroconference, ESA Special Publication, 477, 361-364, 2002

Papoulis, A.: Probability, random variables, and stochastic processes. McGraw-Hill, New York, 1965.

Parks, G. K., Lee, E., Mozer, F., Wilber, M., Lucek, E., Dandouras, I., Rème, H., Mazelle, C., Cao, J. B., Meziane, K., Goldstein, M. L., and Escoubet, P.: Larmor radius size density holes discovered in the solar wind upstream of Earth's bow shock, Phys. Plasmas, 13, 050701, doi:10.1063/1.2201056, 2006.

Paschmann, G. and Daly, P. W.: Analysis methods for multispacecraft data, ESA Publications Division, The Netherlands, 2000.

Rème, H., Aoustin, C., Bosqued, J. M., et al.: First multispacecraft ion measurements in and near the Earths magnetosphere with the identical Cluster ion spectrometry (CIS) experiment, Ann. Geophys., 19, 1303-1354, 2001, http://www.ann-geophys.net/19/1303/2001/. 
Sahraoui, F.: Diagnosis of magnetic structures and intermittency in space-plasma turbulence using the technique of surrogate data, Phys. Rev. E, 78, 026402, doi:10.1103/PhysRevE.78.026402, 2008.

Samsonov, A. A., Alexandrova, O., Lacombe, C., Maksimovic, M., and Gary, S. P.: Proton temperature anisotropy in the magnetosheath: comparison of 3-D MHD modelling with Cluster data, Ann. Geophys., 25, 1157-1173, 2007, http://www.ann-geophys.net/25/1157/2007/.

Schwartz, S. J.: Shocks: commonalities in solar-terrestrial chains, Space Sci. Rev., 124, 333-344, doi:10.1007/s11214-006-9093-y, 2006.

Smith, C. W., L'Heureux, J., Ness, N. F., Acuña, M. H., Burlaga, L. F., and Scheifele, J.: The ACE magnetic fields experiment, Space Sci. Rev., 86, 613-632, 1998.

Smith, C. W., Hamilton, K., Vasquez, B. J., and Leamon, R. J.: Dependence of the dissipation range spectrum of interplanetary magnetic fluctuations on the rate of energy cascade, Astrophys. J. Lett., 645, L85-L88, 2006.

Stasiewicz, K., Longmore, M., Buchert, S., Shukla, P. K., Lavraud, B., and Pickett, J.: Properties of fast magnetosonic shocklets at the bow shock, Geophys. Res. Lett., 30(24), 2241, doi:10.1029/2003GL017971, 2003.
Telloni, D., Antonucci, E., Bruno, R., and D'Amicis, R.: Persistent and self-similar large-scale density fluctuations in the solar corona, Astrophys. J., 693, 1022-1028, doi:10.1088/0004637X/693/1/1022, 2009.

Veltri, P.: MHD turbulence in the solar wind: self-similarity, intermittency and coherent structures, Plasma Phys. Control. Fusion, 41, A787-A795, 1999.

Veltri, P. and Mangeney, A.: Scaling laws and intermittent structures in solar wind MHD turbulence, Solar Wind Nine, pp. 543546, 1999.

Welch, P.: The use of fast Fourier transform for the estimation of power spectra: a method based on time averaging over short, modified periodograms, IEEE Trans. Audio and Electroacoust, AU-15(2), 70-73, 1967.

Zhou, Y., Matthaeus, W. H., and Dmitruk, P.: Magnetohydrodynamic turbulence and time scales in astrophysical and space plasmas, Rev. Mod. Phys., 76, 1015-1035, 2004. 